Proceedings of the Estonian Academy of Sciences,

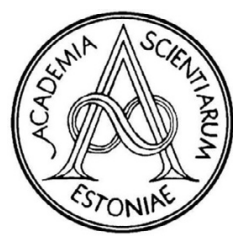
2021, 70, 2, 190-206

https://doi.org/10.3176/proc.2021.2.08

Available online at www.eap.ee/proceedings

POWER QUALITY ANALYSIS

\title{
Bivariate stochastic model of current harmonic analysis in the low voltage distribution grid
}

\author{
Muhammad Naveed Iqbal ${ }^{\mathrm{a} *}$, Lauri Kütt ${ }^{\mathrm{a}}$, Kamran Daniel ${ }^{\mathrm{a}, \mathrm{c}}$, Marek Jarkovoi ${ }^{\mathrm{a}}$, Bilal Asad ${ }^{\mathrm{a}, \mathrm{b}}$ and \\ Noman Shabbir ${ }^{\mathrm{a}}$
}

\begin{abstract}
${ }^{a}$ Department of Power Engineering and Mechatronics, Tallinn University of Technology, Ehitajate tee 5, 19086 Tallinn, Estonia
${ }^{\mathrm{b}}$ Department of Electrical Engineering and Automation, Aalto University, 02150 Espoo, Finland

${ }^{c}$ Department of Electrical, Electronics and Telecommunication Engineering, University of Engineering and Technology (FSD Campus), Lahore, Pakistan
\end{abstract}

Received 4 January 2021, accepted 31 March 2021, available online 6 May 2021

(c) 2021 Authors. This is an Open Access article distributed under the terms and conditions of the Creative Commons AttributionNonCommercial 4.0 International License (http://creativecommons.org/licenses/by-nc/4.0/).

\begin{abstract}
This paper presents a bottom-up bivariate analysis approach to estimate current harmonics by taking account of network and load variations. The current harmonics assessment in the presence of existing and future nonlinear loads is vital to study their impact on the distribution grid. The traditional harmonic analysis models consider only stable loads while neglecting the harmonic interaction among the devices. Modern nonlinear loads operate under different working modes and configurations. Thermal stability, harmonic cancellation, and dynamic network parameters influence the current harmonic estimations. In this paper, a probabilistic approach is presented to model harmonic emission in the low voltage distribution grid under network and load uncertainties. A case study is used to demonstrate effectiveness of the proposed model.
\end{abstract}

Key words: probabilistic assessment, network uncertainties, current harmonics, load modelling.

\section{INTRODUCTION}

Current harmonic emission is becoming a critical power quality issue as the uptake of nonlinear devices in the distribution grid is increasing. Rampant electricity demand, fluctuating fuel prices, and greenhouse gas (GHG) emission is pushing the manufacturer of electrical equipment toward energy-efficient design. Consequently, all modern household appliances incorporate electronic-based converters. These devices draw non-sinusoidal currents and cause voltage distortion in the distribution grid. High penetration of electric vehicles and smart buildings will further increase the share of nonlinear devices in the coming years. The primary task of the network operators is to maintain power quality within the limits for the network and, therefore, a realistic estimation of current harmonics is critical. This paper presents an overview of a probabilistic approach to estimate current harmonics in the low voltage (LV) distribution grid in the presence of different uncertainties.

The power quality indicates an aggregated effect of electromagnetic disturbances that can degrade the voltage and current waveforms. One of the critical parameters of power quality is the sine waveform of the

\footnotetext{
*Corresponding author, miqbal@taltech.ee
} 
network voltage. The periodic variations from the sinewave, characterized as harmonics, are responsible for the voltage waveform deterioration. As the share of these voltage harmonics increases, the reliability of the network is imperiled. The current and voltage distortions may lead to several problems in the distribution network. The incremental higher frequencies amplify the proximity and skin effects in the cables. The performance and life span of transformers, cables, and other network components may be reduced because of the added stress [1,2]. The network protection equipment and electrical appliances can malfunction due to these unwanted harmonics [3]. Furthermore, the neutral conductor can be overloaded and it leads to undesirable consequences in the network [4]. The capacitor banks can also fail as the higher frequencies can alter their impedance [5]. Therefore, details about harmonic sources and their effect on the network are vital for the network operators to understand the power system's smooth operation within the limits of power quality indices.

Although sufficient literature is available related to power flow modelling, nonlinear load modelling and stochastic current harmonic estimations are relatively contemporary. The classical residential load models are developed to predict energy consumption patterns and are, therefore, unable to estimate current harmonic emissions mainly because of their low time resolution. Due to the stochastic nature of the modern nonlinear devices, it is not easy to model their usage and operational behaviour. Typically, a general overview is presented based on assumptions and limited measurements of the harmonic currents. On the other hand, several uncertainties are associated with a probabilistic approach for harmonic estimation. Domestic electrical appliances can operate in different modes and the harmonic emission profile could be very different for each mode. Thermal stability associated with electronic devices causes variation in the current harmonics, leading to an inaccurate assessment of power quality indices [6]. The harmonic cancellation also occurs as voltage or current harmonics are aggregated by geometrical vector addition because of the phase angles associated with them [7]. As a result, the aggregated harmonic content at the point of common coupling (PCC) may increase or decrease [8]. The assumptions and uncertainties linked with harmonic analysis lead to an inefficient modelling approach with inaccurate harmonic estimations.

This paper provides a framework of probabilistic modelling of current harmonics with nonlinear loads in the distribution network. The model relies on comprehensive power quality measurements and a probabilistic approach to model the harmonic current magnitude and phase angles. The electrical appliance usage patterns were developed to evaluate a nonlinear device's impact on the grid by the residential occupants. The load measurements were performed at different voltage waveforms, including sine wave, to observe the harmonic cancellation impact on harmonic aggregation. The harmonic analysis approach at different voltage levels will provide an insight into the real-time effect of electronic load on the network. The network impedance provides the way to calculate harmonic emission limits and is often estimated by using short circuit impedance [9]. The modern electronic load contains additional passive components that may affect the network impedance. The current harmonic measurements of electronic loads with different voltage waveforms will enable us to estimate the change in the load's impedance at various frequencies. Figure 1 shows how to estimate the change in network impedance when the electronic load is connected at the point of

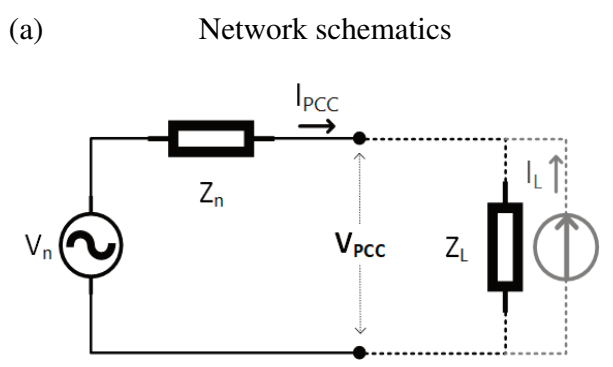

(b) Waveform of current and voltage

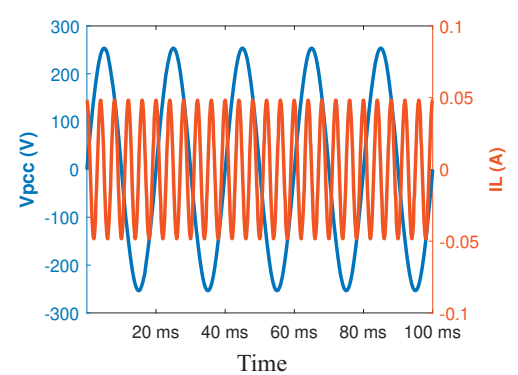

Fig. 1. Current and voltage waveforms at the PCC. 
common coupling at a particular frequency. In the first stage, the voltage $V_{P C C}$ and the current $I_{P C C}$ from the network at the PCC are measured before connecting the load. The current $I_{L}(150 \mathrm{~Hz})$ is then injected and new measurements of the voltage $V_{P C C}^{\prime}$ and the current $I_{P C C}^{\prime}$ are taken at the PCC. The network impedance can be detected at the frequency $f$ by using Eq. 1:

$$
Z_{f}=\frac{V_{P C C}^{\prime}-V_{P C C}}{I_{P C C}^{\prime}-I_{P C C}}
$$

The existing harmonic estimation methods are presented in Section 2. Accuracy and uncertainties are analysed in Section 3 and Section 4 describes the proposed modelling approach for current harmonic estimation. A case study used to evaluate harmonic emission from the lighting load in the distribution grid is introduced in Section 5. Conclusions are presented in Section 6.

\section{HARMONIC ESTIMATION METHODS}

The distribution network is designed to provide sinusoidal voltage to the consumers. The modern nonlinear loads distort the voltage by adding current harmonics. A detailed harmonic analysis could provide insight into the power system behaviour under these voltage and current distortions. Researchers have made different models to estimate the current harmonics for the residential network. These models can be classified into time or frequency domain equivalent load models or measurement-based models.

The frequency-domain models are easier to compute and consider the frequency domain attributes of the electrical equipment. The simplest frequency domain model is the current source model based on the current magnitude and phase measurement of each harmonic under consideration. Any nonlinear load can be represented by the sum of constant current sources for each frequency [10]. Each current harmonic source is independent of the input voltage. However, in the distribution grid, the voltage distortions can alter the harmonic emissions of electrical equipment [11]. As the voltage waveform in a distribution network changes continuously depending on the type and amount of the connecting load, current source models are not effective for harmonic analysis. The Norton model addresses this problem by considering the admittance matrix. The model parameters are estimated by switching the operating condition of the power system with the assumption that the impedance and current of the Norton model will be constant and will not change with the shape of the voltage waveform. Two different sets of current and voltage harmonics are used to estimate the model parameters by means of Eq. 2 and Eq. 3:

$$
\begin{gathered}
Z_{n, h}=\frac{V_{h}^{\prime}-V_{h}}{I_{h}^{\prime}-I_{h}}, \\
I_{n, h}=\frac{V_{h}}{Z_{h, k}}-I_{h} .
\end{gathered}
$$

Here, $Z_{n, h}$ and $I_{n, h}$ are the impedance and current of the Norton equivalent model for the harmonic $h$ calculated by using two sets of current and voltage measurements $V_{h}, I_{h}$ and $V_{h}^{\prime}, I_{h}^{\prime}$. Although this approach provides some advantages over the current source model, it is unable to consider the cross dependency of harmonics. The harmonic currents only depend on the corresponding voltage harmonics and not on the voltage waveform itself.

This shortcoming was improved by employing a crossed frequency admittance matrix in the Norton coupled (NC) model. The estimated harmonic currents using this model will depend not only on the voltage harmonics of the same frequency but also on the other frequencies. Eq. 4 and Eq. 5 represent the mathematical form of the NC model. The verification and comparison of frequency-domain models are presented in $[12,13]$.

$$
\vec{I}=\vec{Y} \times \vec{V}
$$




$$
\left[\begin{array}{c}
\vec{I}_{1 m} \\
\vec{I}_{2 m} \\
\vec{I}_{3 m} \\
\ldots \\
\vec{I}_{n m}
\end{array}\right]=\left[\begin{array}{ccccc}
\vec{Y}_{11} & \vec{Y}_{12} & \vec{Y}_{13} & \ldots & \vec{Y}_{1 m} \\
\vec{Y}_{21} & \vec{Y}_{22} & \vec{Y}_{23} & \ldots & \vec{Y}_{2 m} \\
\vec{Y}_{31} & \vec{Y}_{32} & \vec{Y}_{33} & \ldots & \vec{Y}_{3 m} \\
\vdots & \vdots & \vdots & \ldots & \vdots \\
\vec{Y}_{n 1} & \vec{Y}_{n 2} & \vec{Y}_{n 3} & \ldots & \vec{Y}_{n m}
\end{array}\right] \cdot\left[\begin{array}{c}
\vec{V}_{1 m} \\
\vec{V}_{2 m} \\
\vec{V}_{3 m} \\
\ldots \\
\vec{V}_{n m}
\end{array}\right]
$$

The time-domain models are based on the load's actual circuits and provide in-depth information about the load harmonic emission profile. A time-domain harmonic analysis approach was applied to nonlinear loads categorized based on their circuit topologies in [11]. Most electronic devices contain switch mode power supplies (SMPSs), for which reason time-domain models equivalent to the SMPS were created. The current harmonic estimation was provided on simulated and measured waveforms. In another study, a harmonic model was formulated for computer loads connected to a single transformer [14]. The results show harmonic cancellation and voltage waveform distortion at the transformer. The mathematical models of low power compact fluorescent lamps (CFLs) were made to study harmonic penetration in [15]. The voltage and current waveforms were recorded and analysed by means of circuit simulation software. Although the time-domain models provide an accurate harmonic analysis approach, they have limited application as it is challenging to model every load connected to the grid using its circuit schematic.

In the electrical model-based approach, the load connected to the network is categorized based on their electrical properties, and the probability distribution of each group is defined to estimate the overall harmonic emission. For example, the network load can be divided into linear or nonlinear devices. These devices can be additionally categorized according to circuit topology and power quality characteristics. A similar probabilistic harmonic analysis model was proposed in 1987 [16]. The model categorized nonlinear loads into four categories based on the switching state and operating mode. The harmonic aggregation analysis was performed using the Monte Carlo approach with probability density functions (PDFs) of harmonic magnitude and phase angles. Based on the appliance measurement data and their usage patterns, a harmonic analysis approach was applied to study the harmonic impacts of the household appliance in the low voltage distribution grid [17]. The results obtained from the harmonic model were then compared with the real-time measurements of the network. A similar bottom-up probabilistic harmonic estimation modelling approach was presented in [18]. The model generated a household appliance's usage patterns based on occupant behaviour, and the appliance's equivalent circuits were used to analyse the harmonic emission. The simulation results of harmonic loads were compared with the actual grid measurement results to extract correlated data. In another study, a probabilistic model to analyse waveform distortions was presented under the influence of high penetration of electric vehicles (EVs). The authors highlight the importance of this approach as uncertainties associated with the EV charging patterns can be easily accounted for [19]. The single and three-phase nonlinear loads were divided into groups based on their current THD (total harmonic distortion) in [20]. The participation of these load groups was obtained based on energy usage patterns at different times of the day. The author selected the customer database parameters by assuming that the data of any particular device type belonged to a normal distribution. The voltage distortion in the low voltage network was evaluated based on this probabilistic method.

In the measurement-based models, current harmonic emission is analysed from the probability distributions of harmonic current measurement data. The measurements could be taken at the electrical appliance level in a bottom-up approach, and aggregated harmonic analysis could provide the harmonic estimation at the point of common coupling. In the top-down approach, measurements are taken at the distribution transformer. In both cases, extensive measurement data is usually compared with an appropriate probability distribution. The voltage distortion in the distribution network was estimated by using Monte Carlo simulation of the aggregated harmonic current in [21]. The measurement data was assumed to fit a normal distribution. The harmonic currents were measured of residential and commercial loads at the point of common coupling in [22]. The measurements were divided into low, medium, and high demand subgroups and compared with the normal distribution and uniform distributions. 
Two different approaches can be applied to construct a probabilistic model based on the type and amount of data [23]. The first approach could be termed as a parametric model where a finite set of data parameters can be compared with predefined distributions. In the second non-parametric approach, the model is based on distributions calculated from the data itself [24]. The parametric models mostly employ the normal distribution defined by mean and variance. The probability density function of a normal distribution is indicated by Eq. 6:

$$
f_{x}=\frac{1}{\sqrt{2 \pi \sigma^{2}}} \times e^{-\frac{(x-\mu)^{2}}{2 \sigma^{2}}} .
$$

Here, $\sigma$ is the standard deviation and $\mu$ represents the mean value of $x$.

In the early harmonic models, the normal distribution was used to describe both magnitude and phase angles as independent variables. However, this assumption is not accurate for harmonic analysis. Therefore, a joint or bivariate probabilistic approach is more effective where the estimated variable depends on the probability density function of two variables. In [10], the load current for residential buildings was estimated using beta bivariate distributions. In [11,12], the joint normal distribution (JNB) was employed for the forecasting of harmonic emissions. The parameters of the normal joint distribution, $\sigma$ (standard deviation) and $\mu$ (mean value) are calculated by Eq. 7 and Eq. 8 using the complex components of the current $i_{x}$ and $i_{y}$ :

$$
\begin{gathered}
\mu_{x y}=\left[\begin{array}{l}
i_{x} \\
i_{y}
\end{array}\right], \\
\sum x y=\left[\begin{array}{cc}
\sigma^{2} i_{x} & \sigma\left(i_{x}, i_{y}\right) \\
\sigma\left(i_{x}, i_{y}\right) & \sigma^{2} i_{y}
\end{array}\right] .
\end{gathered}
$$

Figure 2a shows the 15th harmonic current when normal distribution fitting parameters are applied in a complex plane for a display monitor. The individual probability density of the real and imaginary parts of the current harmonics is indicated by red and blue lines, respectively. The green circle encloses the part of the distribution responsible for 95 percentile of the estimated values. Although the joint probability distribution provides better results than the individual normal distribution for $x$ and $y$ values, it can, however, be effective only when both components are linearly dependent. The nonlinear devices with multiple operating modes result in different harmonic currents. The resultant distribution fit of these devices could be very different from the normal distribution. Figure $2 b$ shows the 9 th harmonic current spread of a personal computer (PC) stress test in a complex plane where three different clusters are clearly visible. The normal joint distribution cannot represent this data efficiently. This problem can be addressed by clustering the data and applying the JNB to respective clusters. This approach is known as a multivariate normal mixture and provides a more flexible distribution fit [25]. The distribution mixture approach was applied in [26] to study power quality impact in low voltage distribution. The PDFs of the current harmonics were calculated by finite normal distribution components with their associated weights. The drawback of this approach is that the model requires predefined cluster information.

An adaptive kernel density estimation (KDE) with a plug-in bandwidth selection approach is presented in [27]. The KDE algorithm designates probability distribution for every data point using a kernel function and bandwidth, also known as the smoothing parameter, indicated in Eq. 9:

$$
f_{h}=\frac{1}{N} \sum_{i=1}^{N} K_{b}\left(h-h_{i}\right)=\frac{1}{N_{b}} \sum_{i=1}^{N} K \frac{\left(h-h_{i}\right)}{b} .
$$

Here, $f_{h}$ provides the PDF of $h$ for $N$ observations. $K$ is the kernel and $b$ is the bandwidth. The sum of kernels provides the total probability density of a variable. The optimal bandwidth selection is critical in a KDE model. A large bandwidth will smooth the probability density curve but results in fewer data points in each kernel. As a result, information about data variation will be lost. The optimal methods for finding bandwidth are introduced in [28]. The KDE algorithm, along with the Monte Carlo simulation, was used to 

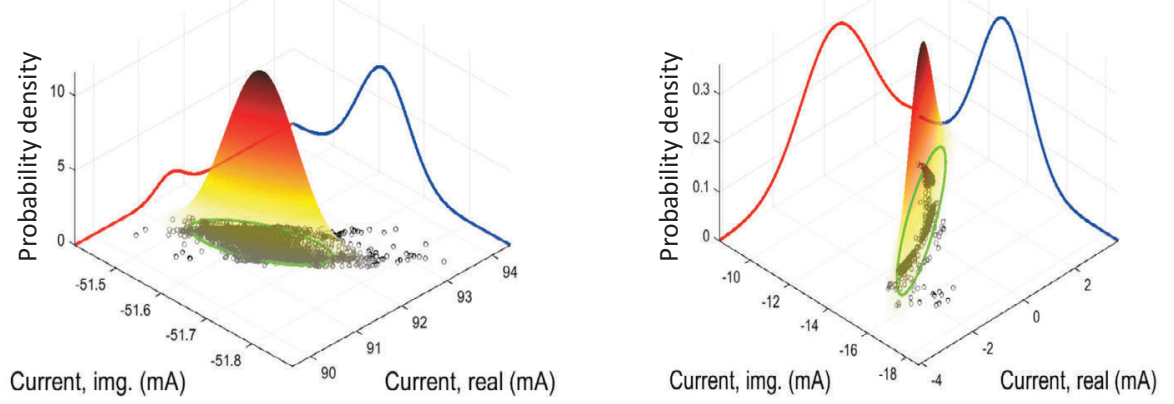

Fig. 2. Joint probability distribution applied to harmonic currents drawn by the monitor and PC [23].

estimate harmonic load flow in [24] and [29]. However, harmonic current magnitude and phase angles were estimated independently, which would provide inaccurate phasor data results. A joint distribution from the KDE algorithm can generate better results where multidimensional vectors represent the parameters. In Fig. 3a, the KDE is applied to the 5th harmonic current measured during the PC stress test. This method requires intensive calculations, and high computational power is needed for even a small-scale harmonic analysis.

Empirical bivariate histogram (EBH) distribution is another approach that divides data into predefined bins. The EBH distribution data is normalized by using Eq. 10 to create a probability density mesh:

$$
P_{x, y}=\frac{C(x, y)}{N \cdot W_{x} \cdot W_{y}}
$$

Here, $P_{x, y}$ is the probability density of a bin at $(x, y), C(x, y)$ are the number of samples in the bin and $W_{x}, W_{y}$ defines the area of the bin. $N$ defines the total number of data points. Figure $3 \mathrm{~b}$ demonstrates the histogram distribution applied to the 5th harmonic current of a PC under stress test. The advantage of EBH over KDE distribution is that it requires less computational power. However, both $\mathrm{EBH}$ and KDE distributions generate unused data space in the PDF when clusters are present in the data. Data sampling for these techniques is quite challenging.

(a)

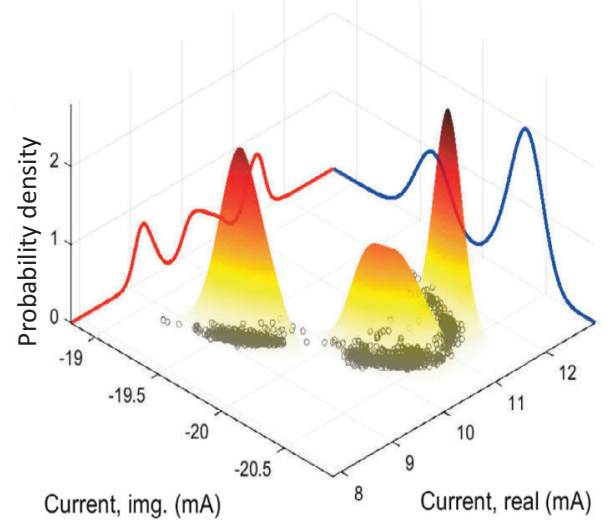

(b)

Histogram distribution

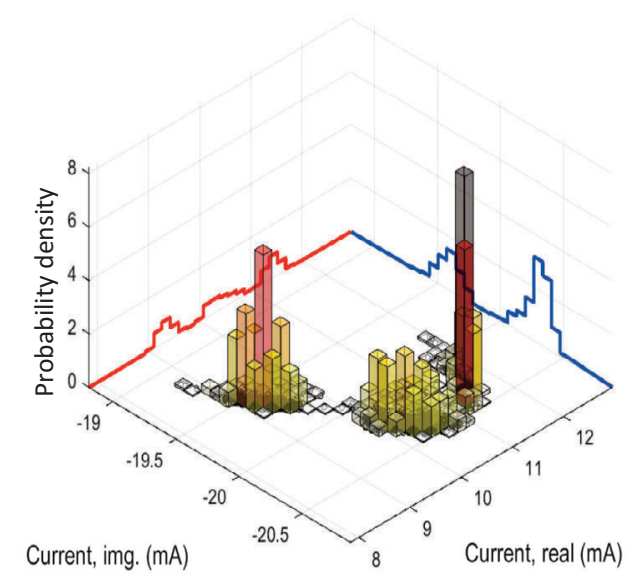

Fig. 3. KDE and histogram distributions applied to the 5th harmonic current of the PC [23]. 


\section{ACCURACY AND UNCERTAINTIES}

Current harmonic emission could be affected by several factors, including network configurations, load variations, appliance parameters, and measurement uncertainties [30]. The power system uncertainties are widely addressed in the literature. The network uncertainty includes variation in supply voltage, frequency, and resonance. They are difficult to model as various factors, including generation, dispatch, and network topologies, affect the estimations [31,32].

The load connected to each bus in the distribution network is comprised of various linear and nonlinear loads. During different times in the day, various loads are connecting and disconnecting to the buses. The researchers use varied stochastic approaches to predict network load behaviour [33,34]. Modern electrical appliances work in various modes that generate different harmonic emission profiles. Different parameters associated with the appliances also vary due to the variations incorporated during the manufacturing process [35]. The environmental conditions and aging of the equipment play their role as well [36].

The measurement uncertainties are associated with the environment, measuring instrument uncertainties, and variations in the test equipment. As the current harmonic profiles vary under different operating modes of the devices, the measurement results should include these variations. The supply voltage distortion also alters the current harmonics of different loads. Therefore, it is challenging to measure specific electrical equipment on different modes under different supply variations. The thermal stability also changes the current harmonics of the electrical appliances. The thermal stability effect on light-emitting diode (LED) lamps shows a significant variation in current harmonics during the stability time. The effect of current harmonic estimation displays a significant error as well [37]. Similarly, power supplies also show a more than 20\% and 12\% variation in the THD and the total RMS current, respectively [38].Likewise, the harmonic current cancellation affects the outcome of harmonic analysis. The higher-order harmonic indicates a more prominent reduction if the harmonic cancellation is taken into account [7]. The harmonic currents are also affected by the cable impedance [39]. Therefore, all of these uncertainties should be included in the model to estimate current harmonic emission.

\section{PROPOSED STOCHASTIC MODELLING APPROACH}

The modern electronic equipment operates in different modes, and their current harmonic spread is irregular with clustered data. The probabilistic approach of modelling current harmonics has the advantage of tackling any sporadic variations. The stochastic models to estimate the current harmonic described in the previous section have several limitations in terms of accuracy or computational complexity. Most of the models use a normal distribution or joint normal distribution fit, not appropriate for most of the current harmonic measurement data as they show different distribution spreads. The KDE and histogram distribution algorithms require a bandwidth selection and become inefficient if the data has clusters.

A novel empirical bivariate probability distribution (EBPD) approach is applied in this research as a part of the proposed bivariate stochastic (BS) model to estimate the current harmonics in the low voltage network. The current harmonic magnitude data is used to generate the empirical cumulative distribution function (ECDF) for the harmonics under consideration. The ECDF will provide the groups of the real and complex components with their probabilities. The model consists of three parts: appliance usage model, measurement database, and empirical bivariate harmonic current model. The algorithm of the model is described with a flow chart in Fig. 4.

The model will simulate the required number of houses for a given number of days to estimate the magnitude and phase angles of the harmonic currents generated by each house appliance. During each day, all houses are populated with the appliance stock, and the current harmonics of every appliance are estimated using the EBPD model. The appliance stock and usage pattern of that appliance are provided by the appliance usage (AU) model further described in this section. Every appliance is simulated individually, and the total harmonic emission of an individual household is aggregated in each iteration. The harmonic 


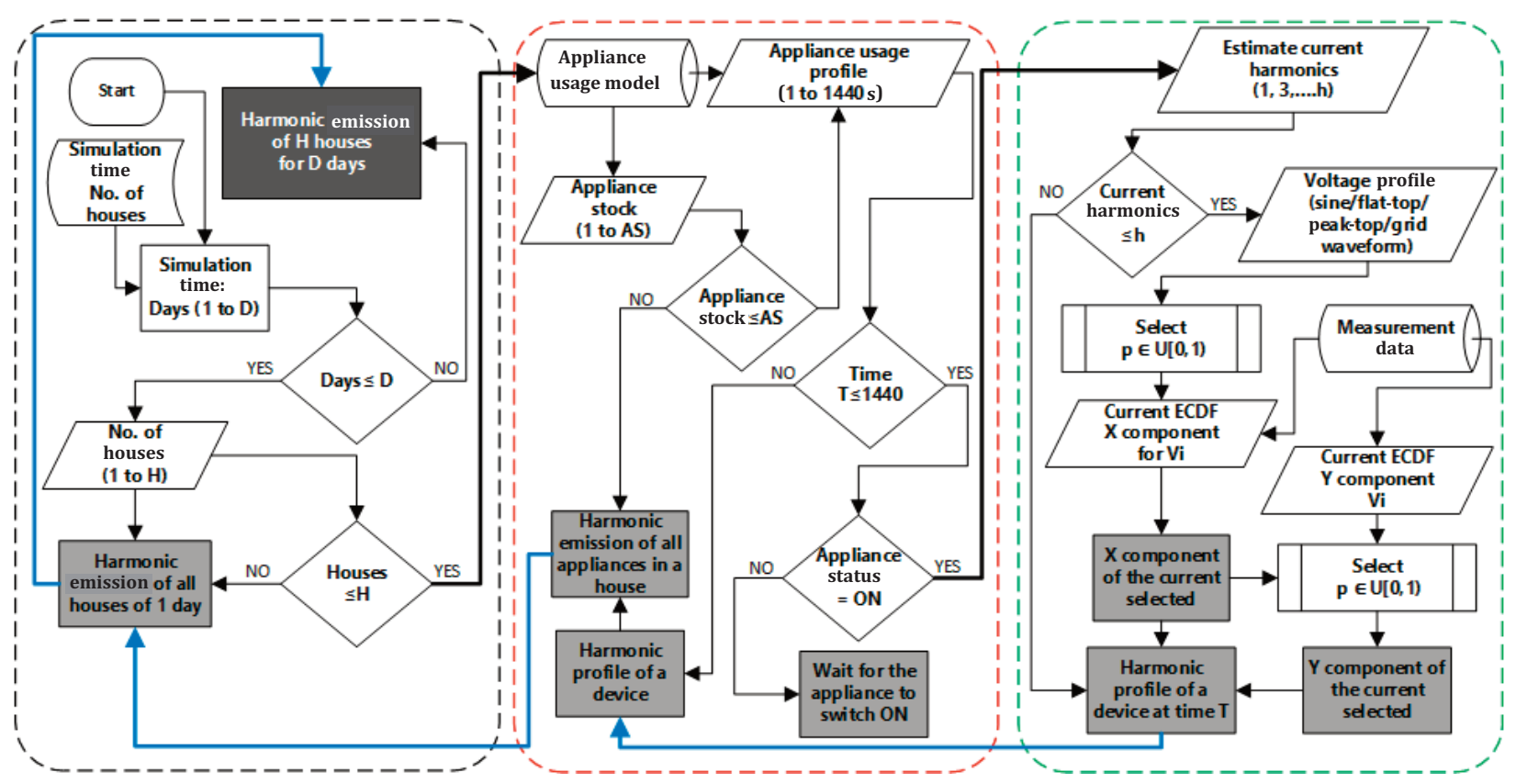

Fig. 4. Flow chart of the proposed model.

currents are aggregated in a complex plane. Therefore, the model also estimates real and complex (X and Y) components of each current harmonic.

\subsection{Appliance usage model}

An efficient current harmonic estimation model should take into account the load variation in the distribution grid. Harmonic injection in a network at a given time depends on the type and amount of the load connected to the grid on that particular instant. However, the load prediction is a complex task as it is difficult to estimate when the consumer is going to use a particular appliance. The occupant behaviour of using electrical appliances is challenging to model as it depends on many factors. Occupants interact with the electrical and nonelectric systems installed in the building, altering thus the energy usage patterns [40]. The International Energy Agency (IEA) also regards occupant actions as the primary cause of controlling the environmental parameters to maintain a comfortable living atmosphere [41]. These occupant actions are responsible for $71 \%$ variation in the building's energy consumption [42]. However, various factors influence the resident's behaviour, including their age, income, social status, and cultural background [43]. The building structure, insulation quality, climate conditions also play their role. Therefore, universal occupancy modelling is near to impossible.

The electricity consumption models can be broadly classified into three categories: top-down models, bottom-up models, and hybrid models [44]. The top-down modelling approach is based on data collected on the macro-level. It may include an electricity billing database, national census, or survey data. The researchers have frequently used the Time Use Survey (TUS) data collected in Europe, Britain, and America for their energy consumption models. These surveys collect data from the targeted groups based on different parameters. The models based on similar data have many drawbacks and lack the capability to provide a detailed analysis of the physical behaviour of the building systems.

The bottom-up models are based on physical measurements at the device or building level. Nevertheless, these models provide accurate information regarding energy consumption in a building but are complicated to construct due to the involvement of several variables. These variables include occupancy, occupant behaviour, climate conditions, building structure, and an extensive database of appliance's measurements. As 
it is difficult to consider each variable in detail, a compromise is required to make a specific model for a particular research problem. Another approach is to combine the benefits of both bottom-up and top-down approaches to improve efficiency. These models are termed as hybrid models [45].

We need a residential electricity consumption model to estimate current harmonic emissions from the building. Therefore, a high-resolution bottom-up model is required to provide usage patterns of domestic appliances that can be compared with the power quality (PQ) measurements. Figure 5 illustrates the abstract diagram of our appliance usage model for residential buildings.

For this study, a residential building in Estonia is measured at the device level for one month. The data is used to construct a probabilistic model to estimate the switching behaviour of the appliance. The model consists of active occupancy profiles, appliance stock in the households, and the electricity consumption measurements as shown in Fig. 5.

Active occupancy profiles are created based on the electricity consumption of the appliance that comes under the direct influence of the occupant's activities. The usage of lighting, media, kitchen, cleaning, and laundry appliances directly depends on the occupant's behaviour. Electricity consumption metre data has been used for occupancy modelling in many studies [46-49]. A similar approach is applied here to create a two state active occupancy profile. ECDFs are created for both weekdays and weekends occupancy status based on the electricity consumption data. A survey related to the occupant's daily activities is also used to improve these occupancy profiles.

Every household has different appliances depending on the family size, geographical location and socioeconomic status. Appliances are also available from different manufacturers with various specifications. Manufacturers introduce new models every year with improved functionality and energy ratings. The appliance ownership information can be extracted from several surveys conducted on national level in different countries. A domestic energy model was created on the basis of a set of common appliances based on national ownership statistics for the United Kingdom (UK) in [50]. Similar surveys are also conducted in Europe and the USA.

The appliance usage model (AU) will provide the information when a particular appliance is switched $\mathrm{ON}$ in the house. The ECDFs are used to generate switching and duration intervals of each appliance in the house. The total electricity consumption of a single housing unit can be determined from the Eq. 11:

$$
E=\sum_{\text {day }=1}^{D}\left[\sum_{a p=1}^{n}\left(P_{a} \times d_{a}\right)+\left(P_{s} \times d_{s}\right)\right] .
$$

Here, $P_{a}$ denotes the active power of the appliance $a p$ in its active mode and $P_{s}$ is its active power in the standby mode. $d_{a}$ and $d_{s}$ are the time duration of an appliance operating in active or standby modes during a day.

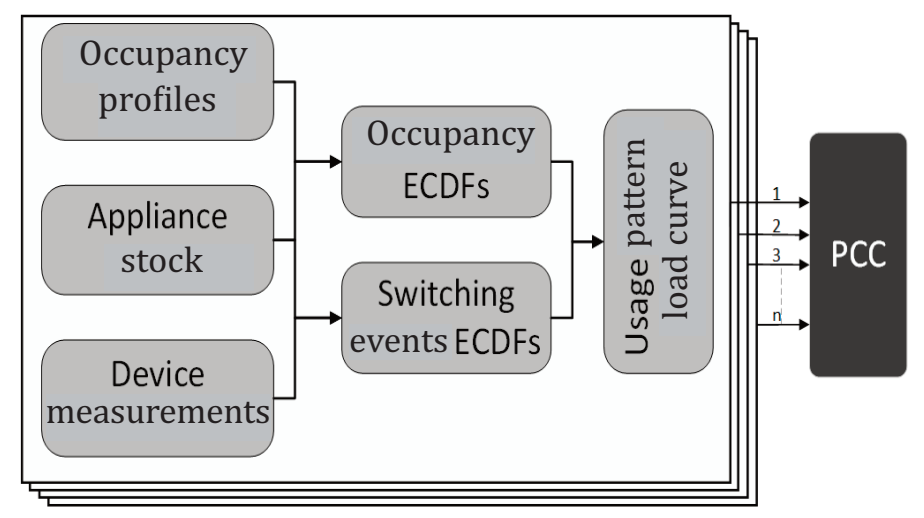

Fig. 5. Abstract diagram of the appliance usage model. 


\subsection{Measurement database}

The measurement database contains the current harmonic measurements of different household appliances operating at different working modes on various voltage waveforms. We have used sinusoidal, peak-top, flat-top, and real-time grid voltage waveforms as an input to measure current harmonics.

For the real-time grid voltage waveforms, a low voltage residential network is measured at 5-minute intervals for a day to record the voltage harmonic magnitude and phase angles. Figure 6 shows the voltage waveform used to measure the current harmonic emission from the household appliances using the measurement test bench.

The measurement test bench consists of a PC with MATLAB program and a data acquisition (DAQ) module from National Instruments to generate a reference signal for the controllable power supply. The reference signal $V_{R}$ has enabled us to generate the required voltage waveform $V_{O}$ using Eq. 12:

$$
V_{O}=\frac{V_{R} \times V_{\text {range }}}{V_{C}} .
$$

Here, $V_{\text {range }}$ is $300 \mathrm{~V}$ and $V_{C}$ is 7.07. $V_{R}$ is generated by using the voltage harmonic magnitudes and phase angles by means of Eq. 13:

$$
V_{\text {out }}=\sum_{i=1}^{n} \sqrt{2} \times A_{i} \sin \left(2 \pi f_{i} t_{s}+\alpha_{i}\right) .
$$

Here, $A_{i}$ represents the root mean square values of the voltage harmonics and $\alpha_{i}$ indicates the phase difference from the fundamental frequency. $f_{i}, t_{s}$ is the harmonic frequency for the ith harmonic and sampling interval, respectively. The sampling frequency of the reference signal is $100 \mathrm{kHz}$ and it is indicated by $f_{s}$. Figure 7 demonstrates the schematic of the measurement setup.

The harmonic current estimation model is based on the power quality measurement data of the appliance portfolio. The device usage patterns from Section 3.1 are compared with the harmonic current profiles of each household appliance. The model can be used to evaluate the total harmonic emission of a multiple house as illustrated in Fig. 8.

\subsection{Empirical bivariate harmonic current model}

The empirical bivariate harmonic current modelling approach is suitable for harmonic analysis of loads with the dynamic profile of harmonic emission and is also capable of addressing different uncertainties responsible

(a) Synthetic voltage waveforms

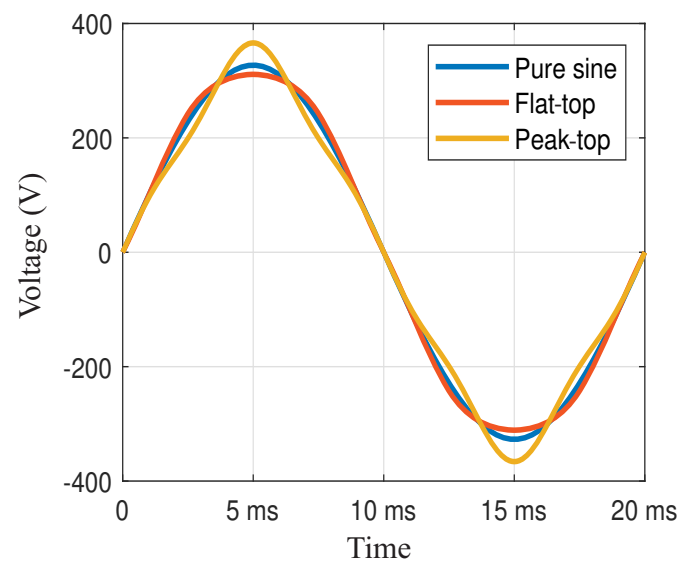

(b)

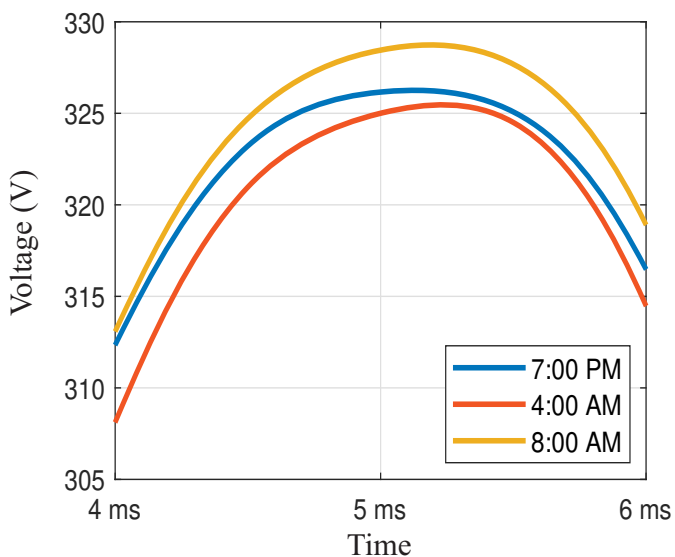

Fig. 6. Voltage waveforms used for current harmonics measurements. 


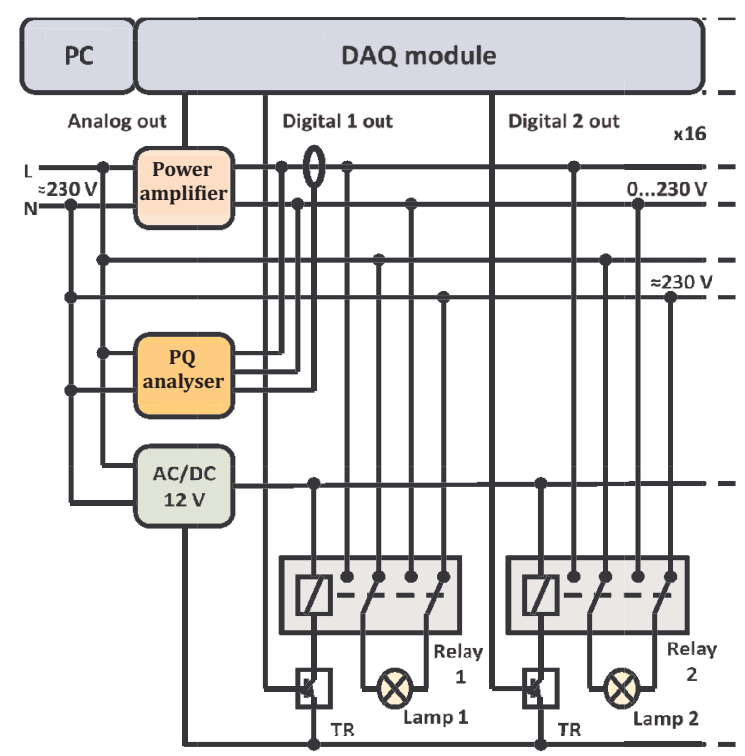

Fig. 7. Schematic of the measurement setup.

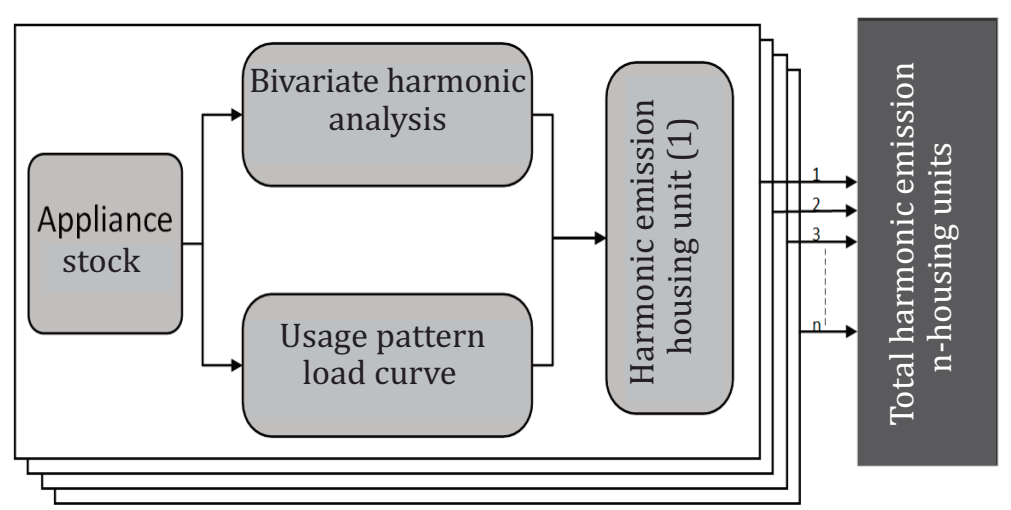

Fig. 8. Harmonic current estimation of multiple households.

for current harmonic variations. The current harmonics of individual appliances will be modelled by means of the ECDF. In the first step, the ECDF for the real part of the current in the complex domain is calculated. Each group of this ECDF is further mapped with the complex part of the variable's data group, and the ECDF of each bivariate data group is calculated. The resolution of the ECDF determines the accuracy of the harmonic estimation model. ECDFs for both real and imaginary components are calculated using Eq. 14:

$$
p_{m}(X \leq x)=\frac{1}{m} \sum_{k=1}^{m} 1\left[x_{k} \leq t\right]
$$

Here, $p_{m}$ is the cumulative probability function of $m$ groups. The 1 is called indicator function and has two possible values as shown by Eq. 15:

$$
1\left[x_{k} \leq t\right]= \begin{cases}1 & \text { for } x_{k} \leq x \\ 0 & \text { for } x_{k}>x\end{cases}
$$

Figure 9 demonstrates how ECDFs of real and imaginary components of the current harmonics can be used to create distribution of the current harmonic in the complex plane. The red line shows the ECDF of the real 


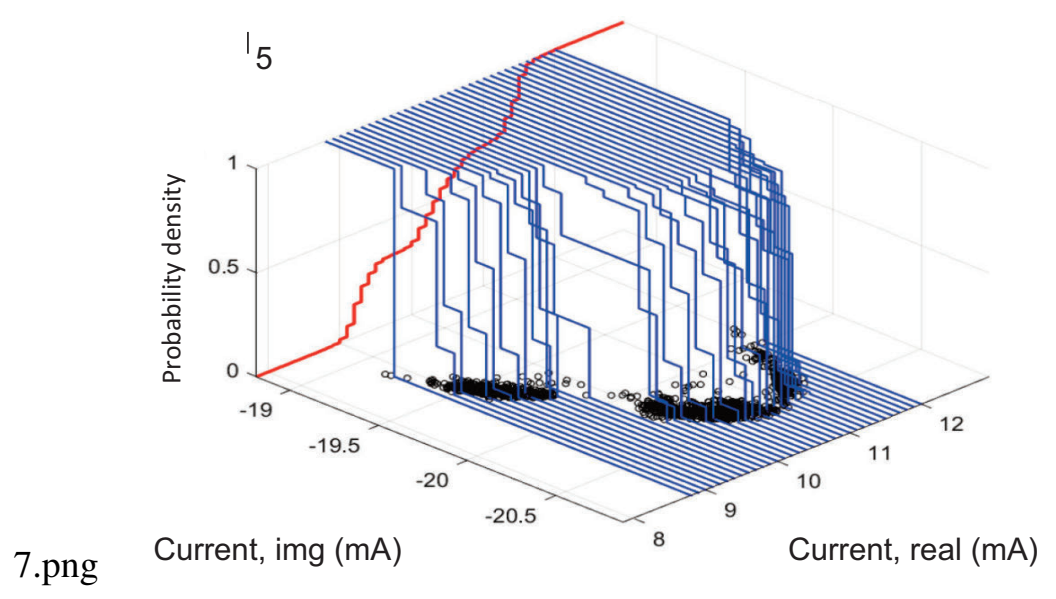

Fig. 9. EBPD applied to the 5th harmonic of the PC under stress test [23].

component of the harmonics and each blue line indicates the ECDF of the imaginary components at each group of the real component's ECDF.

\section{CASE STUDY OF HARMONIC ESTIMATIONS}

The proposed bivariate stochastic model was used to estimate current harmonic emission from the lighting load in the low voltage residential grid. The lighting usage profiles were made utilizing the AU model with a 1-minute resolution. The lighting measurements from the measured residential building were analysed for this purpose. The active occupancy profiles and electricity consumption data were the inputs of our lighting usage model. Total lighting load was segregated into the usage profile of each switch to control the electrical lights in the building. The load curves were divided into morning, day, and evening cycles. Each cycle was simulated separately.

The ECDFs were created to generate the probability distribution function of the switching and noise events. The switching events occur when a lamp is turned $\mathrm{ON}$ for more than 10 minutes. All events with a lamp usage of less than 10 minutes are considered noise events. The time duration of switching and noise events for all lamps in a house can be aggregated to find the total lighting power demand. Equation 16 can be used to calculate the total energy consumed by each lamp during any cycle in a residential building:

$$
E_{l}=\sum_{t=1}^{T}\left[\sum_{s e=1}^{m}\left(d_{s e} \times P_{l}\right)+\sum_{n e=1}^{n}\left(d_{n e} \times P_{l}\right)\right] .
$$

Here, $E_{l}$ is the total energy consumed by a lamp in one cycle of $T$ minutes duration with $m$ and $n$ switching and noise events, respectively. $d_{s e}$ refers to the duration of each switching event and $d_{n e}$ is the duration of one noise event. The 60 houses were simulated for 100 days, and each house was populated with different LED lamps depending on the lumens needed in each room. The usage pattern from the AU model provided the time at which a particular lamp would switch ON as well as the ON time duration. A Monte Carlo based approach was applied to calculate the current harmonics injected by the lighting from all the 60 houses. The lamps operate in a single working mode (ON or OFF state) if dimming circuits are not used. Therefore, bivariate harmonic estimation is simplified. The lamps were measured on different voltage waveforms. These waveforms were regenerated using the measurement test bench described in Section 4.3. Lamps were warmed up for 1 hour to eliminate the measurement variation because of thermal instability. The voltage waveforms included sinusoidal, peak-top, flat-top, and real grid waveforms during different times of a day. The probabilities were assigned to each voltage waveform, as shown in Table 1. 
Table 1. Waveform probability used in the model

\begin{tabular}{|l|c|}
\hline Waveform & Probability \\
\hline Sinusoidal & 0.15 \\
\hline Flat-top & 0.06 \\
\hline Peak-top & 0.04 \\
\hline Grid waveform 1 & 0.25 \\
\hline Grid waveform 2 & 0.25 \\
\hline Grid waveform 3 & 0.25 \\
\hline
\end{tabular}

After selecting the voltage waveform during each iteration, the real components of the current harmonics were selected for the particular voltage waveform. In the next step, the complex component of the current harmonics was generated based on the group assigned to the selected real component. The real and imaginary components of current harmonics for all lamps were generated for a day with 1-second resolution. Figure 10 illustrates the total RMS current drawn by the lighting usage of 60 houses. The bold black line shows the mean value of the RMS current, and the blue dotted line indicates the 90 percentile value of the RMS current consumed by the lighting in 60 houses. The minimum value of the RMS current drawn by the lighting usage in all 60 houses is illustrated by the red dotted line.

The high-frequency current harmonics can also be estimated in a similar process. Figure 11 shows the 3rd, 5th, 7th, and 9th current harmonic represented by black, red, blue, and green colours, respectively. The bold line indicates the mean value, and the dotted line shows the 90th percentile value.

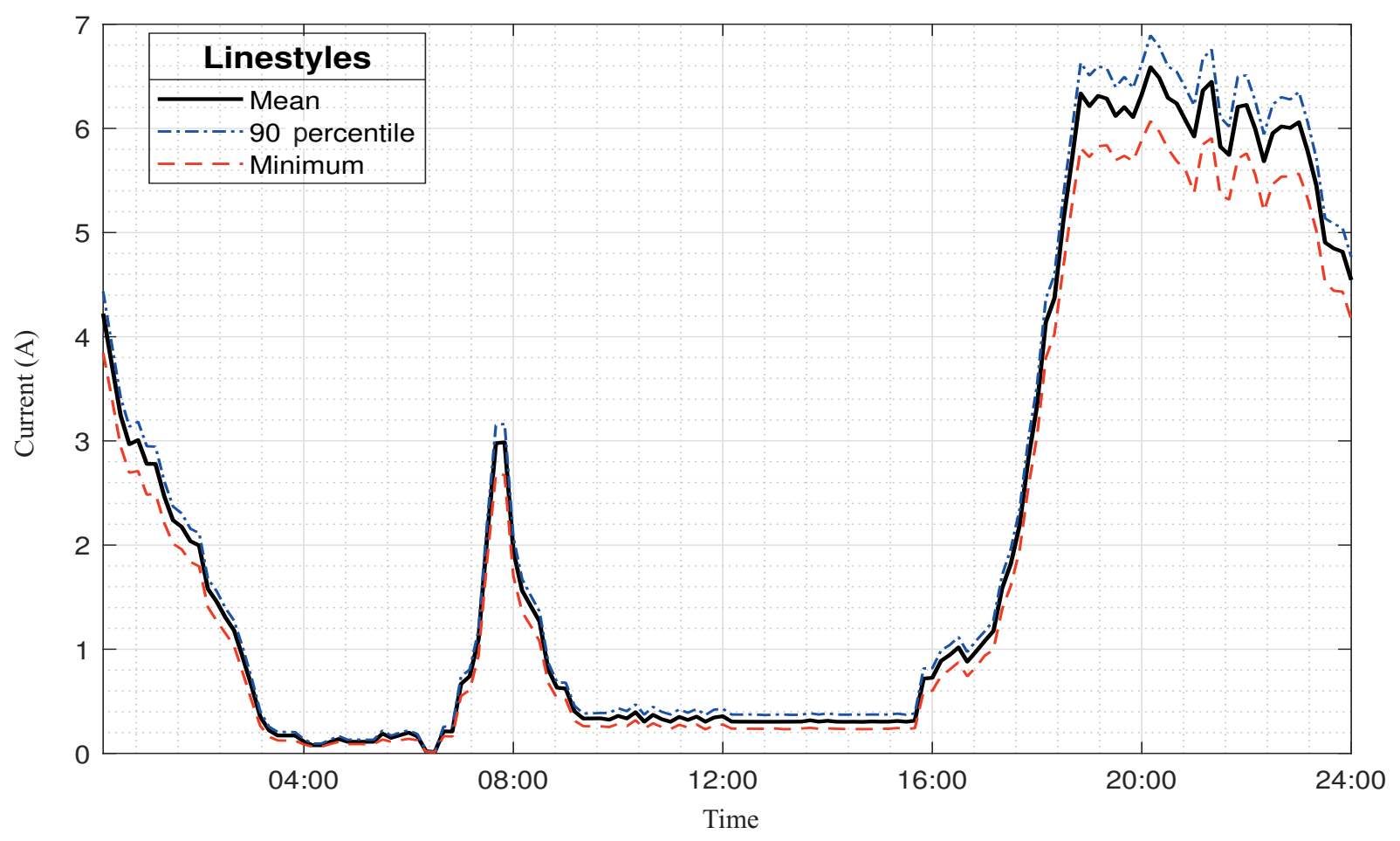

Fig. 10. Total RMS current estimated for the lighting load of 60 houses for 100 days. 


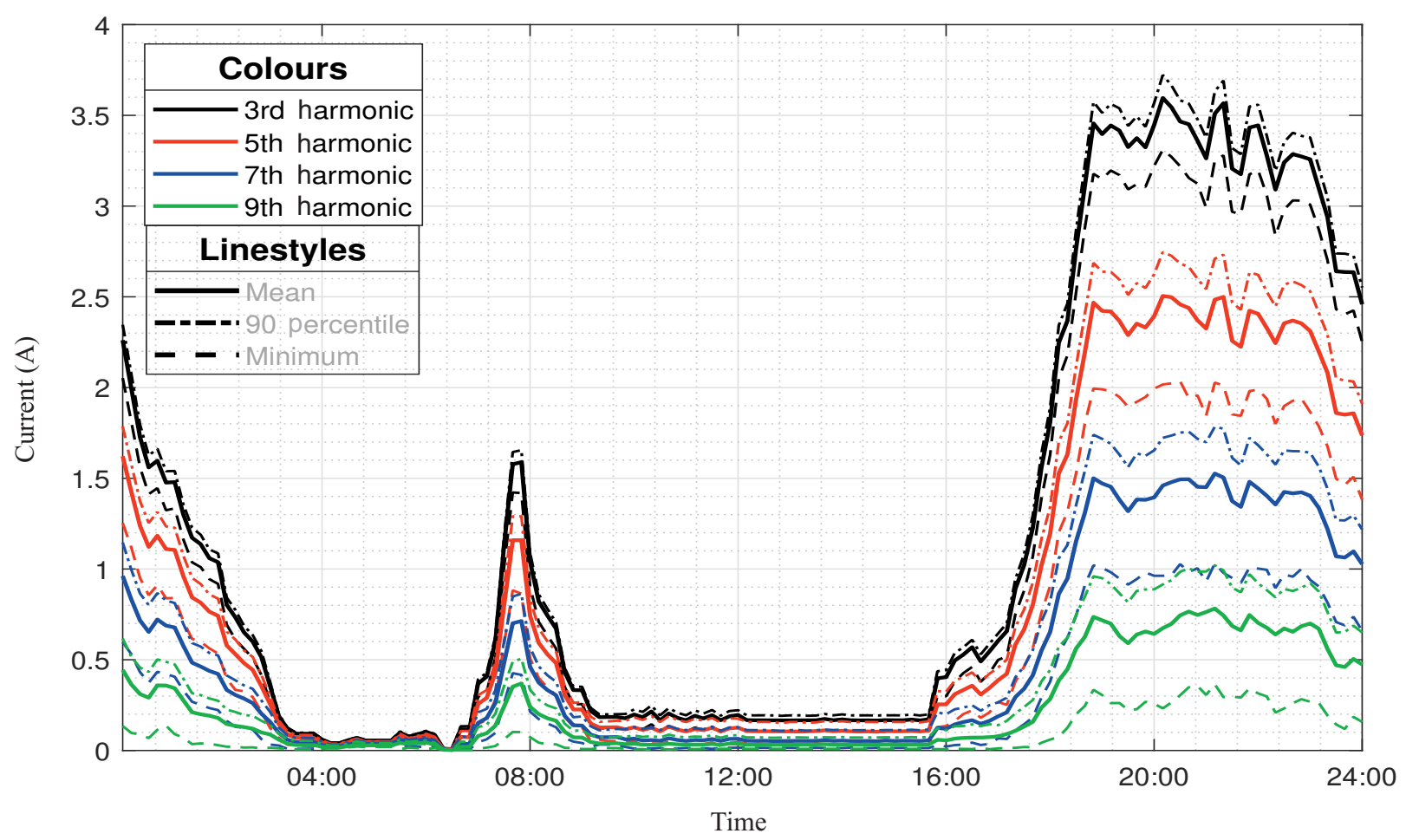

Fig. 11. Harmonic current estimation of the lighting load of 60 houses for 100 days.

The results reveal the effectiveness of the proposed approach as it is simple to evaluate, and any uncertainty could be added at any step. The model provides flexibility to evaluate present and future appliances by expanding the measurement database with power quality measurements of devices on different voltage waveforms.

\section{CONCLUSIONS}

A novel bottom-up stochastic model to assess the current harmonic emission is presented in this paper. The bivariate empirical distribution approach is used to model harmonic currents. It provides a simple and flexible option to evaluate different aspects of harmonic emission in the distribution grid. The model is capable of handling any uncertainty associated with the distribution grid by considering its stochastic nature.

The stochastic approach has made the model more efficient in handling variations and uncertainties than the traditional numerical or probabilistic methods. The bivariate approach is applied to model current harmonics, making the model capable of processing data with high variations and clusters.

An appliance usage model is also presented based on the real-time measurements at the device level. The occupancy and appliance stock data can be used to create usage profiles of different electrical appliances in a residential building. Thermal stability, cable impedance, and grid side variation are considered during the measurements. The model is flexible to include additional future loads such as electric vehicles. It provides an accurate assessment of the power quality aspects from the perspective of the low voltage distribution under dynamic load and network conditions. The case study provides the effectiveness of the model by estimating the current harmonic emission due to the lighting load of sixty houses. The measurement database could be extended in the future by including measurements of different household appliances. 


\section{ACKNOWLEDGEMENTS}

This work was supported by the Estonian Research Council grant PSG 142. The publication costs of this article were covered by the Estonian Academy of Sciences.

\section{REFERENCES}

1. Pierce, L. W. Transformer design and application considerations for nonsinusoidal load currents. IEEE Trans. Ind. Appl., 1996, 32(3), 633-645.

2. Shareghi, M., Phung, B. T., Naderi, M. S., Blackburn, T. R. and Ambikairajah, E. Effects of current and voltage harmonics on distribution transformer losses. In Proceedings of the IEEE International Conference on Condition Monitoring and Diagnosis, Bali, Indonesia, September 23-27, 2012. IEEE, 2013, 633-636.

3. Czarnecki, L. S. Comments on active power flow and energy accounts in electrical systems with nonsinusoidal waveforms and asymmetry. IEEE Trans. Power Delivery, 1996, 11(3), 1244-1250.

4. Watson, N. R., Scott, T. L. and Hirsch, S. J. J. Implications for distribution networks of high penetration of compact fluorescent lamps. IEEE Trans. Power Delivery, 2009, 24(3), 1521-1528.

5. Clement-Nyns, K., Haesen, E. and Driesen, J. The impact of charging plug-in hybrid electric vehicles on a residential distribution grid. IEEE Trans. Power Syst., 2010, 25(1), 371-380.

6. Iqbal, M. N., Jarkovoi, M., Kütt, L. and Shabbir, N. Impact of LED thermal stability to household lighting harmonic load current modeling. In Proceedings of Electric Power Quality and Supply Reliability Conference $(P Q)$ \& Symposium on Electrical Engineering and Mechatronics (SEEM), Kärdla, Estonia, June 12-15, 2019. IEEE, 2019, 1-6.

7. Jarkovoi, M., Iqbal, M. N. and Kütt, L. Analysis of harmonic current stability and summation of LED lamps. In Proceedings of Electric Power Quality and Supply Reliability Conference (PQ) \& Symposium on Electrical Engineering and Mechatronics (SEEM), Kärdla, Estonia, June 12-15, 2019. IEEE, 2019, 18957598.

8. Hansen, S., Nielsen, P. and Blaabjerg, F. Harmonic cancellation by mixing nonlinear single-phase and three-phase loads. IEEE Trans. Ind. Appl., 2000, 36(1), 152-159. https://doi.org/10.1109/28.821810

9. Chakravorty, D., Meyer, J., Schegner, P., Yanchenko, S. and Schocke, M. Impact of modern electronic equipment on the assessment of network harmonic impedance. IEEE Trans. Smart Grid, 2017, 8(1), 382-390.

10. Henao-Muñoz, A. C. and Saavedra-Montes, A. J. Comparison of two mathematical models for nonlinear residential loads. In Proceedings of the 17th International Conference on Harmonics and Quality of Power (ICHQP), Belo Horizonte, Brazil, October 16-19, 2016.

11. Blanco, A. M., Yanchenko, S., Meyer, J. and Schegner, P. Impact of supply voltage distortion on the current harmonic emission of non-linear loads. DYNA, 2015, 82(192), 150-159.

12. Koch, A. S., Myrzik, J. M. A., Wiesner, T. and Jendernalik, L. Evaluation and validation of Norton approaches for nonlinear harmonic models. In Proceedings of IEEE Grenoble Conference PowerTech, Grenoble, France, June 16-20, 2013. IEEE, 2013, $1-6$.

13. Almeida, C. F. M. and Kagan, N. Harmonic coupled norton equivalent model for modeling harmonic-producing loads. In Proceedings of the 14th International Conference on Harmonics and Quality of Power-ICHQP 2010, Bergamo, Italy, September 26-29, 2010. IEEE, 2010, 1-9.

14. Ahmed, E. E., Xu, W. and Zhang, G. Analyzing systems with distributed harmonic sources including the attenuation and diversity effects. IEEE Trans. Power Delivery, 2005, 20(4), 2602-2612. https://doi.org/10.1109/TPWRD.2005.855441

15. Cunill-Sola, J. and Salichs, M. Study and characterization of waveforms from low-watt $(<25 \mathrm{~W})$ compact fluorescent lamps with electronic ballasts. IEEE Trans. Power Delivery, 2007, 22(4), 2305-2311.

16. Baghzouz, Y. and Tan, O. T. Probabilistic modeling of power system harmonics. IEEE Trans. Ind. Appl., 1987, IA-23(1), 173180.

17. Ye, G., Nijhuis, M., Cuk, V. and Cobben, J. F. G. Stochastic residential harmonic source modeling for grid impact studies. Energies, 2017, 10(3), 372. https://doi.org/10.3390/en10030372

18. Salles, D., Jiang, C., Xu, W., Freitas, W. and Mazin, H. E. Assessing the collective harmonic impact of modern residential loadsPart I: methodology. IEEE Trans. Power Delivery, 2012, 27(4), 1937-1946. https://doi.org/10.1109/TPWRD.2012.2207132

19. Caramia, P., Proto, D., Russo, A. and Varilone, P. Probabilistic harmonic analysis for waveform distortion assessment of low voltage distribution systems with plug-in hybrid electric vehicles. In Proceedings of the 1st International Conference on Energy Transition in the Mediterranean Area (SyNERGY MED), Cagliari, Italy, May 28-30, 2019. IEEE, 2019, 1-6.

20. $\mathrm{Au}, \mathrm{M}$. T. and Milanović, J. V. Establishing harmonic distortion level of distribution network based on stochastic aggregate harmonic load models. IEEE Trans. Power Delivery, 2007, 22(2), 1086-1092. https://doi.org/10.1109/TPWRD.2007.893193

21. Au, M. T. and Milanović, J. V. Stochastic assessment of harmonic distortion level of medium voltage radial distribution network. In Proceedings of the 9th International Conference on Probabilistic Methods Applied to Power Systems, Stockholm, Sweden, June 11-15, 2006. IEEE, 2007, 1-6.

22. Au, M. T. and Milanović, J. V. Development of stochastic aggregate harmonic load model based on field measurements. IEEE Trans. Power Delivery, 2007, 22(1), 323-330. https://doi.org/10.1109/TPWRD.2006.881455

23. Jarkovoi, M., Kütt, L. and Iqbal, M. N. Probabilistic bivariate modeling of harmonic current. In Proceedings of the 19th International Conference on Harmonics and Quality of Power (ICHQP), Dubai, United Arab Emirates, July 6-7, 2020. IEEE, 2020, 1-6. 
24. Nasrfard-Jahromi, F. and Mohammadi, M. Probabilistic harmonic load flow using an improved kernel density estimator. International Journal of Electrical Power and Energy Systems, 2016, 78, 292-298. https://doi.org/10.1016/j.ijepes.2015.11.076

25. Ray, S. and Lindsay, B. G. The topography of multivariate normal mixtures. Ann. Stat., 2005, 33(5), 2042-2065. https://doi.org/10.1214/009053605000000417

26. Meyer, J. and Schegner, P. Characterization of power quality in low voltage networks based on modeling by mixture distributions. In Proceedings of the 9th International Conference on Probabilistic Methods Applied to Power Systems, PMAPS, Stockholm, Sweden, June 11-15, 2006. IEEE, 2007.

27. Botev, Z. I., Grotowski, J. F. and Kroese, D. P. Kernel density estimation via diffusion. Ann. Statist., 2010, 38(5), $2916-2957$. https://doi.org/10.1214/10-AOS799

28. Wȩglarczyk, S. Kernel density estimation and its application. ITM Web of Conferences, 2018, 23, 00037. https://doi.org/10.1051/itmconf/ 20182300037

29. Nasrfard-Jahromi, F. and Mohammadi, M. A sampling-based method using an improved nonparametric density estimator for probabilistic harmonic load flow calculation. Turk. J. Elec. Eng. Comp. Sci., 2016, 24, 51113-5123. https://doi.org/10.3906/elk-1505-197

30. Li, Z., Hu, H., Wang, Y., Tang, L., He, Z. and Gao, S. Probabilistic harmonic resonance assessment considering power system uncertainties. IEEE Trans. Power Delivery, 2018, 33(6), 2989-2998.

31. Sainz, L. and Balcells, J. Harmonic interaction influence due to current source shunt filters in networks supplying nonlinear loads. IEEE Trans. Power Delivery, 2012, 27(3), 1385-1393.

32. Barmada, S., Musolino, A., Raugi, M. and Tucci, M. Analysis of power lines uncertain parameter influence on power line communications. IEEE Trans. Power Delivery, 2007, 22(4), 2163-2171. https://doi.org/10.1109/TPWRD.2007.900305

33. Preece, R. and Milanović, J. V. Efficient estimation of the probability of small-disturbance instability of large uncertain power systems. IEEE Trans. Power Systems, 2016, 31(2), 1063-1072.

34. Abu-Hashim, R., Burch, R., Chang, G., Grady, M., Gunther, E., Halpin, M. et al. Test systems for harmonics modeling and simulation. IEEE Trans. Power Delivery, 1999, 14(2), 579-587. https://doi.org/10.1109/61.754106

35. Morales, J. M. and Pérez-Ruiz, J. Point estimate schemes to solve the probabilistic power flow. IEEE Trans. Power Syst., 2007, 22(4), 1594-1601. https://doi.org/10.1109/TPWRS.2007.907515

36. Pinceti, P. and Prando, D. Sensitivity of parallel harmonic filters to parameters variations. Int. J. Electr. Power Energy Syst., $2015,68,26-32$.

37. Iqbal, M. N., Kütt, L., Asad, B., Shabbir, N. and Rasheed, I. Time-dependent variations in current harmonic emission by LED lamps in the low-voltage network. Electr. Eng., 2020, 101(25), 1277-1293.

38. Iqbal, M. N., Kütt, L., Asad, B., Vaimann, T., Rassõlkin, A. and Demidova, G. L. Time dependency of current harmonics for switch-mode power supplies. Appl. Sci., 2020, 10(21), 7806.

39. Iqbal, M. N. and Lauri, K. Impact of cable impedance on the harmonic emission of LED lamps. In Proceedings of the 21 st International Scientific Conference on Electric Power Engineering (EPE), Prague, Czech Republic, October 19-21, 2020. IEEE, 2020, 1-5.

40. Sun, K., Yan, D., Hong, T. and Guo, S. Stochastic modeling of overtime occupancy and its application in building energy simulation and calibration. Build. Environ., 2014, 79, 1-12. http://dx.doi.org/10.1016/j.buildenv.2014.04.030

41. Yoshino, H., Hong, T. and Nord, N. IEA EBC annex 53: Total energy use in buildings-analysis and evaluation methods. Energy Build., 2017, 152, 124-136. https://doi.org/10.1016/j.enbuild.2017.07.038

42. Sonderegger, R. C. Movers and stayers: The resident's contribution to variation across houses in energy consumption for space heating. Energy Build., 1978, 1(3), 313-324.

43. Feng, X., Yan, D. and Hong, T. Simulation of occupancy in buildings. Energy Build., 2015, 87, 348-359. http://dx.doi.org/10.1016/j.enbuild.2014.11.067

44. Iqbal, M. N. and Kütt, L. End-user electricity consumption modelling for power quality analysis in residential building. In Proceedings of the 19th International Scientific Conference on Electric Power Engineering (EPE), Brno, Czech Republic, May 16-18, 2018. IEEE, 2018, 1-6.

45. Ofetotse, E. L., Essah, E. A. and Yao, R. Domestic energy models: complexities in defining specific tools. In Proceedings of the International Conference of SuDBE2013, Chongqing, China, October 25-28, 2013.

46. Causone, F., Carlucci, S., Ferrando, M., Marchenko, A. and Erba, S. A data-driven procedure to model occupancy and occupantrelated electric load profiles in residential buildings for energy simulation. Energy Build., 2019, 202, 109342. https://doi.org/10.1016/j.enbuild.2019.109342

47. Tekler, Z. D., Low, R. and Blessing, L. Using smart technologies to identify occupancy and plug-in appliance interaction patterns in an office environment. IOP Conf. Ser.: Mater. Sci. Eng., 2019, 609(6), 062010.

48. Molina-Markham, A., Shenoy, P., Fu, K., Cecchet, E. and Irwin, D. Private memoirs of a smart meter. In BuildSys'10: Proceedings of the 2nd ACM Workshop on Embedded Sensing Systems for Energy-Efficiency in Buildings, Zurich, Switzerland, November 2, 2010. ACM, New York, NY, 2010, 61-66.

49. Kleiminger, W., Beckel, C. and Santini, S. Household occupancy monitoring using electricity meters. In UbiComp 2015: Proceedings of the ACM International Joint Conference on Pervasive and Ubiquitous Computing, Osaka, Japan, September 7-11, 2015. ACM, New York, NY, 2015, 975-986.

50. Richardson, I., Thomson, M. and Infield, D. A high-resolution domestic building occupancy model for energy demand simulations. Energy Build., 2008, 40(8), 1560-1566. 


\title{
Madalpinge-jaotusvõrgus esinevate vooluharmoonikute kahemõõtmeline stohhastiline modelleerimine
}

\author{
Muhammad Naveed Iqbal, Lauri Kütt, Kamran Daniel, Marek Jarkovoi, Bilal Asad ja \\ Noman Shabbir
}

On esitatud kahemõõtmeline stohhastiline analüüs vooluharmoonikute hindamiseks alt-üles viisil, mis arvestab võrgu ja koormuste muutumist. Vooluharmoonikute hindamine praeguste ja tulevikus rakedatavate mittelineaarsete koormustega on oluline, et määrata nende mõju jaotusvõrgule. Traditsioonilised harmoonikute analüüsi mudelid arvestavad ainult püsivaid koormusi ja jätavad kõrvale harmoonikute omavahelise mõju. Soojuslik stabiilsus, harmoonikute tühistamine ja võrgu dünaamilised parameetrid mõjutavad samuti vooluharmoonikute hinnanguid. Antud artiklis on esitatud tõenäosuslik lähenemine, millega modelleerida vooluharmoonikute emissiooni madalpinge-jaotusvõrkudes võrgu ja koormuse määramatuse korral. Esitatud mudeli tõhusust on näidatud juhtumipõhise analüüsiga. 